\title{
TACKLING REFRAMING: THE DEVELOPMENT AND EVALUATION OF A PROBLEM REFRAMING CANVAS
}

\author{
Mensch, Sabine Liana; Gonçalves, Milene
}

TU Delft

\begin{abstract}
The problems of today are increasingly complex in nature. Yet, most support provided by design agencies and innovation consultancies is based on unstructured approaches, guided by the facilitators own intuition. This article presents the Teckel canvas, an approach to guide problem reframing in facilitation to both tackle solution and problem understanding. After synthesising the main insights from prominent reframing methods and models, a meta-process of reframing was created, which was translated into a practical canvas. Two Dutch case studies are examined in which the proposed reframing canvas is tested. When this canvas was used in facilitation, we found out that the medium and message can be reframed separately or together and that the canvas can be used as a tool for overview on the subject. Our findings indicate that an explicit and tangible use of reframing can improve solving complex problems to be more clear, visual and reflective.
\end{abstract}

Keywords: Design methods, Design practice, Reframing, Facilitation, Visualisation

\section{Contact:}

Mensch, Sabine Liana

TU Delft

Industrial Design Engineering

The Netherlands

sabine.mensch@hotmail.com 


\section{INTRODUCTION}

The world is continuously changing, and design practices are changing with it. The problems we face today in society and business are complex, extended and connected (Dorst, 2015) and require a different approach, different from simple ideation to implement products in the market. An emergent approach in consultancy is to solve these complex problems by facilitation (Aguirre, Agudelo, and Romm, 2017). Design agencies and innovation consultancies, such as Flatland (in the Netherlands) or Xplane (in the US) have learned that visual thinking together with facilitation, supports framing problems to be able to understand and tackle them (Flatland, 2018a).

\subsection{Definitions of framing, reframing \& co-evolution}

In the context of this paper, we define framing as clarifying and crystallizing a problem or situation (Maister, Green and Galford, 2000). This may consist of drafting hypotheses, problem statements, points of view and determining the relevance of certain issues. As such, the aim of framing is to organise and reveal tacit and relevant knowledge surrounding a client's problem, to get to its root, and to establish a shared understanding of the problem. Once this frame is established, designers and facilitators are in a better position to tackle the (redefined) problem.

At times, reframing of an existing view of the problem is needed, especially when the current frame becomes detrimental to its resolution. Reframing refers then to the process of changing and reflecting on a frame to create new insights or a new frame of meaning (Benammar, 2012). Reframing is an extremely effective approach to solve problems in design practice and facilitation sessions (Paton and Dorst, 2011), especially when there is misalignment in a project or in the absence of an acceptable solution; when the original frame is too complex, too vague, not focused (on the target group or strengths of the company), or not addressing the deeper issues of the problem at hand (e.g., Flatland, 2018a, Dorst, 2015). By accepting that the problem definition is not yet fixed in the beginning of a project and that one can go back and forward into redefining it, reframing is a phenomenon closely related to co-evolution (Maher, Poon and Boulanger, 1996; Dorst and Cross, 2001, Wiltschnig, Christensen and Ball, 2013). In the co-evolution model, the problem and solution spaces evolve together to form iteratively new understandings - or frames - of what the problem may entail, and subsequently, its solutions.

However, despite being a seemingly essential skill in designers (e.g., Paton and Dorst, 2011), it remains unclear how one should go about in order to reframe. Current methods and approaches, those that touch upon reframing, focus mostly on ideation of opportunities, whilst the world's complexity asks for a collaborative exploration of the problem. There is a lack of clear and structured approaches to reframe and visually decode complex problems. Design agencies and consultancies are learning as they go to create their own practices of reframing complexity, but many do not have the opportunity to reflect or structure their process yet (Flatland, 2018a). Nevertheless, more and more companies are requesting reframing services, in order to align their frontstage behaviour with the backstage decision making and actions. Yet, that can only happen if the backstage team has a clear story, reason and plan of change (Sanden and Flipse, 2016). Therefore, this paper investigates reframing as a means to form a different understanding of the problem, supported by visual thinking.

\subsection{New proposition of a problem focused reframing approach}

In this paper, we propose a new reframing method that is focused on collaborative investigation on the co-evolution of the problem and solution space to be better able to explore and solve complex problems. In other words, this reframing method focusses on rethinking the problem and its components to form a problem and solution direction together, within a team, stakeholders, or clients. By changing the focus from solutions to the actual problem, our method can potentially first create an alternative understanding of the starting point to be able to change the way it is perceived, so that the created solutions can be more explicit, visual and reflective. The reflective component can be achieved when the facilitator is more consciously aware of the whole reframing process to see where they started and where they are going. Next to that, the aim of problem reframing is to avoid creating temporary or makeshift solutions, but rather solve problems at their core. 


\subsection{Structure of the paper}

The paper is structured in four parts: After introducing the major concepts we deal with, we move on to review current literature on framing in design research and relevant fields to set the tone for the development of the proposed reframing method in section 2. Section 3 describes a practical method to support problem reframing in facilitation sessions. Section 4 describes the testing of this method with two practical cases. The final sections are reserved to the discussion of our findings, in relation to implications for literature, education and practice.

\section{LITERATURE REVIEW}

Framing is not a new design skill. Since the influential work of Schön (1983), framing has been acknowledged as a necessary step in the design process, not only to fully explore the problem space but also as a way to disassociate from the problem as given.

\subsection{Bridging frames}

The creation of a new frame is usually triggered by surprises, which could include new information, design activities or interaction with team members and stakeholders, for instance (Cash and Gonçalves, 2017). Those surprises do not necessarily need to be game-changing events, but anything that can cause puzzlement and confusion and do not fit the current frame (Stompff, Smulders, and Henze, 2016). Once a surprise is embraced, it may evolve into a new frame, by reflecting on alternate routes. Making analogies and mental simulations, for instance, help to deal with uncertain, complex and ambiguous problems and triggers creativity in conjunction with surprises (Wiltschnig et al., 2013; Stompff, Smulders, and Henze, 2016). According to Schön (1983) and Stompff, Smulders, and Henze (2016), reflection is a necessary step in order to incorporate surprises in a new frame. Designers usually deal with problems that are highly complex because they involve latent needs, desires and experiences of intertwined interests of multiple stakeholders (Dorst, 2015). Reframing the problem could be articulated as an experimental, yet not final, bridge between the problem and solution spaces (Dorst and Cross, 2001).

\subsection{Comparing 8 reframing approaches}

To contextualise this paper within the array of existing models and methods that address reframing, we reviewed the work of the following authors: Benammar (2012), De Jong and van Dijk (2015), De Bono (1984), Dorst (2015), Hey, Joyce and Beckman (2007), Stompff, Smulders, and Henze (2016), Wedell-Wedellsborg (2017), and the ViP method from Hekkert and van Dijk (2011). The aforementioned methods and models were selected as the eight most prominent reframing methods or approaches in the field of design and facilitation. Benammar (2012) and De Jong and van Dijk (2015) both have methods that resembles each other based on forming a redefined main belief, which is the basis for new insights. However, this method is mostly focused on generating new ideas and lacks an in-depth analysis of the main problem itself. In this way, they do not question their starting point. From a pragmatic approach of creativity, De Bono (1984) used creative techniques to challenge the status quo in ideation. In his method "Asking Why", he goes deeper into the problem, yet there is no follow up plan to structure or implement the newly generated insights. Dorst (2015) established a method - frame creation - for an extended investigation of the problem to find the objectives behind a problem, which has been applied in highly complex societal problems. The 9 steps approach of frame creation (Dorst, 2015) relies on the identification of paradoxes, but lacks concrete steps to resolve these necessary contradictions. Hey, Joyce and Beckman (2007) have constructed an underlying understanding of how reframing works in conversation in design teams. However, their model remains theoretical and does not address how one should move from one phase to the next. Likewise, Stompff, Smulders, and Henze (2016) described how reframing occurs in design practice in the "wild", by formulating the underlying features of the reframing process. They distinguish two specific phases, sensemaking and future framing, which are triggered by surprises. Again, they do not elaborate on how to navigate the reframing process purposefully. Wedell-Wedellsborg (2017) has a very practical approach to reframe, which requires an outsider perspective in order to be employed. The new perspective relies on only an outsider. Thus, instead of considering a wide angle of possible alternative directions, only one alternative direction based on the opinion of one is implemented. Hekkert and van 
Dijk (2011) developed the ViP approach (Vision in Product Design), which states that in order to think of a future product, one should start by establishing its reason to exist. Their creative approach is solution driven, by focussing on the future situation, even though they recognise that the problem needs to be reformulated. Combining the information above, we see that there is a need for a structured approach to facilitate reframing, by exploring the needs and experiences within the problem itself, and to allow for surprises and reflection. There is not yet an approach that incorporates these features while guiding the reframing of the situation or problem as opposed to reframing for ideation. Likewise, it is also important to consider how reframing can be facilitated in practice. On this regard, visual thinking can be extremely important to increase explicitness and tangibility to reframing (Calabretta, Gemser and Karpen, 2016).

Therefore, the research question is the following: How can we support problem reframing in facilitation sessions, in order to achieve reflective, explicit and visual outcomes in reframing?

\section{METHOD}

In order to develop a practical method to support problem reframing in facilitation sessions, an iterative design process, during the development of a master graduation project, took place within the daily practices of a design consultancy specialised in visual thinking - Flatland. Flatland's services include visually creating visions, strategies or new ways of working, in order to untangle complexity and engage organisations into action. Thus, they are often confronted with the need to reframe the original problems their clients put forward. This design consultancy was then quite appropriate to explore the development of a systematic and practical method to support problem reframing. Following the review presented earlier, we now move on to distil the main insights retrieved from the methods/approaches of Benammar (2012), De Jong, and van Dijk (2015), De Bono (1984), Dorst (2015), Hey, Joyce and Beckman (2007), Stompff, Smulders, and Henze, (2016), Wedell-Wedellsborg (2017), and the ViP method from Hekkert and van Dijk (2011). The creation of the reframing method was as followed:

- The eight aforementioned approaches were refined into their different elements. This included, for instance, the idea of "main beliefs" from Benammar (2012) or considering the "history" from Dorst's "Archeology" perspective (2015);

- To those distilled elements, we added a number of design principles, which were not previously considered in the 8 reframing approaches. Together, all elements were clustered into four categories: 1) Foundation, 2) Analysis \& Question, 3) Challenge \& Focus, and 4) Direction (figure 1).

The result is a meta-overview of what should be considered during a reframing facilitation session the reframing process. For each category, there are subcategories, which are indicated and visualised in figure 1 (under goals). This meta-overview was not yet a practical shortlist of the necessary components that need to be dealt with during a reframing session, so a new iteration was made. This resulted into the Teckel Canvas - a practical tool that can be used during a facilitation session (figure 2). The canvas went through recurrent iterations with a team of professional facilitators from Flatland to improve the visual guidance, facilitation possibilities and consultancy considerations. The Teckel canvas was divided into the same four categories, rewritten into practical steps. They were: 1) Look (which corresponds to 'Foundation'); 2) Touch ('Analysis \& Questions'); 3) Kick ('Challenges \& Focus'); and finally, 4) Direct ('Direction'). The categories were renamed and visually represented to follow the metaphor of a Teckel dog (Teckel is the Dutch translation for the dog breed Dachshund, and it is used as a wordplay for "tackle" the problem). Each category was divided in two sub categories: 1a) Situation, 1b) Context, 2a) Feelings \& Dilemma, 2b) Motivation, 3a) Alternatives \& Inspiration, 3b) Choices, 4a) Goal, and 4b) How to get there (see figure 2). The blocks in figure 2 symbolise the reframing stages. In the first stage, the situation and context are gathered by 'looking' and 'sniffing'. The second stage concerns discovering what individual frames and motivation may exist (represented by the 'heart' and 'gut feeling'). In the Kick stage, challenges and focus become explicit and first ideas appear ('kicking the ball'). Lastly, the Direct stage is about action, where paths to achieve certain goals are set to form a solution direction (the 'tail' sets a direction). After each stage of the canvas, the facilitator should strive to form insights that summarise the essence of each phase. Summarising insights eliminates non-important details, pushes decisions and sets the focus for the next step. It is important to note that the Teckel reframing canvas provides guidance but it is not strict. 
It is possible to move in between steps and back and forward, as long as it is done in a structured way and the facilitator is capable to handle it.
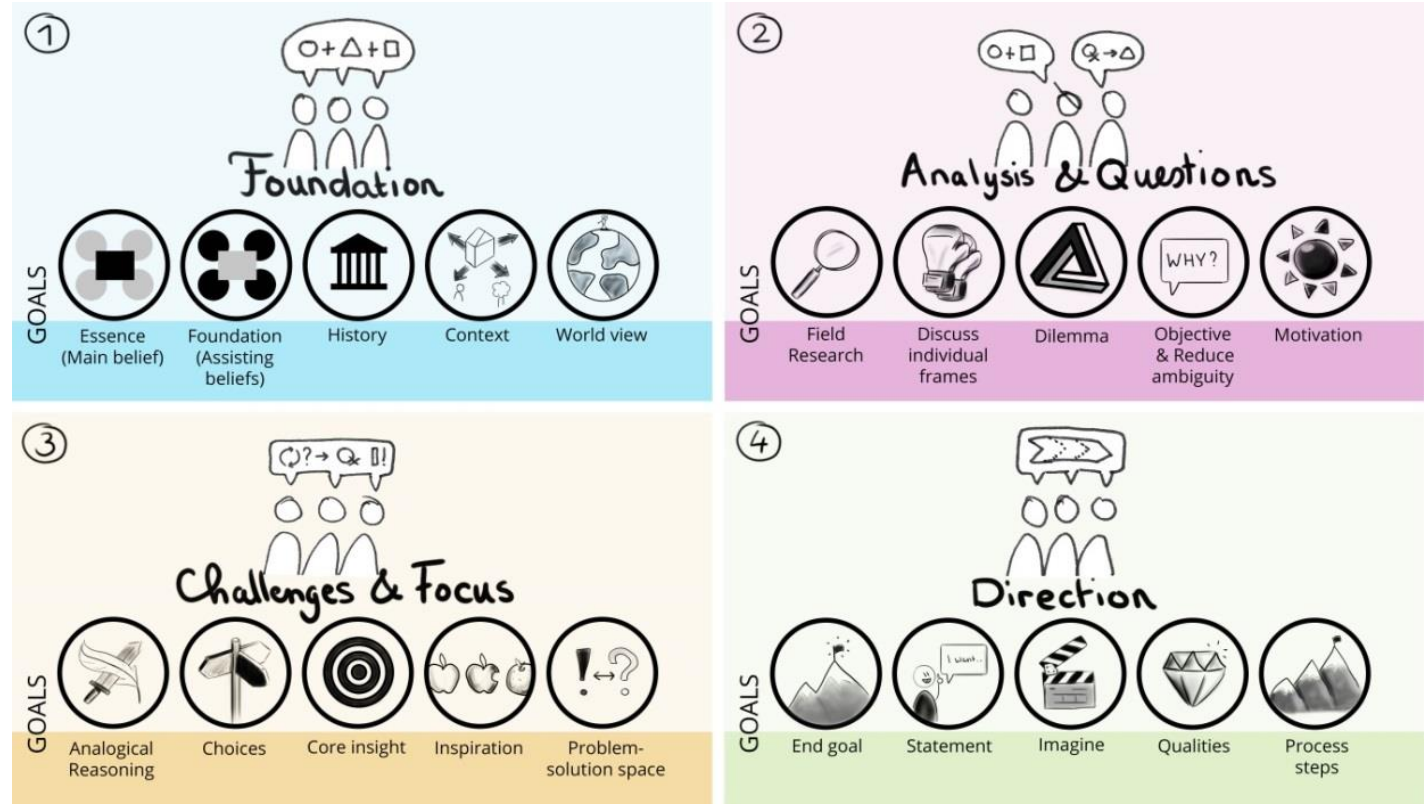

Figure 1. The meta-overview of the reframing process

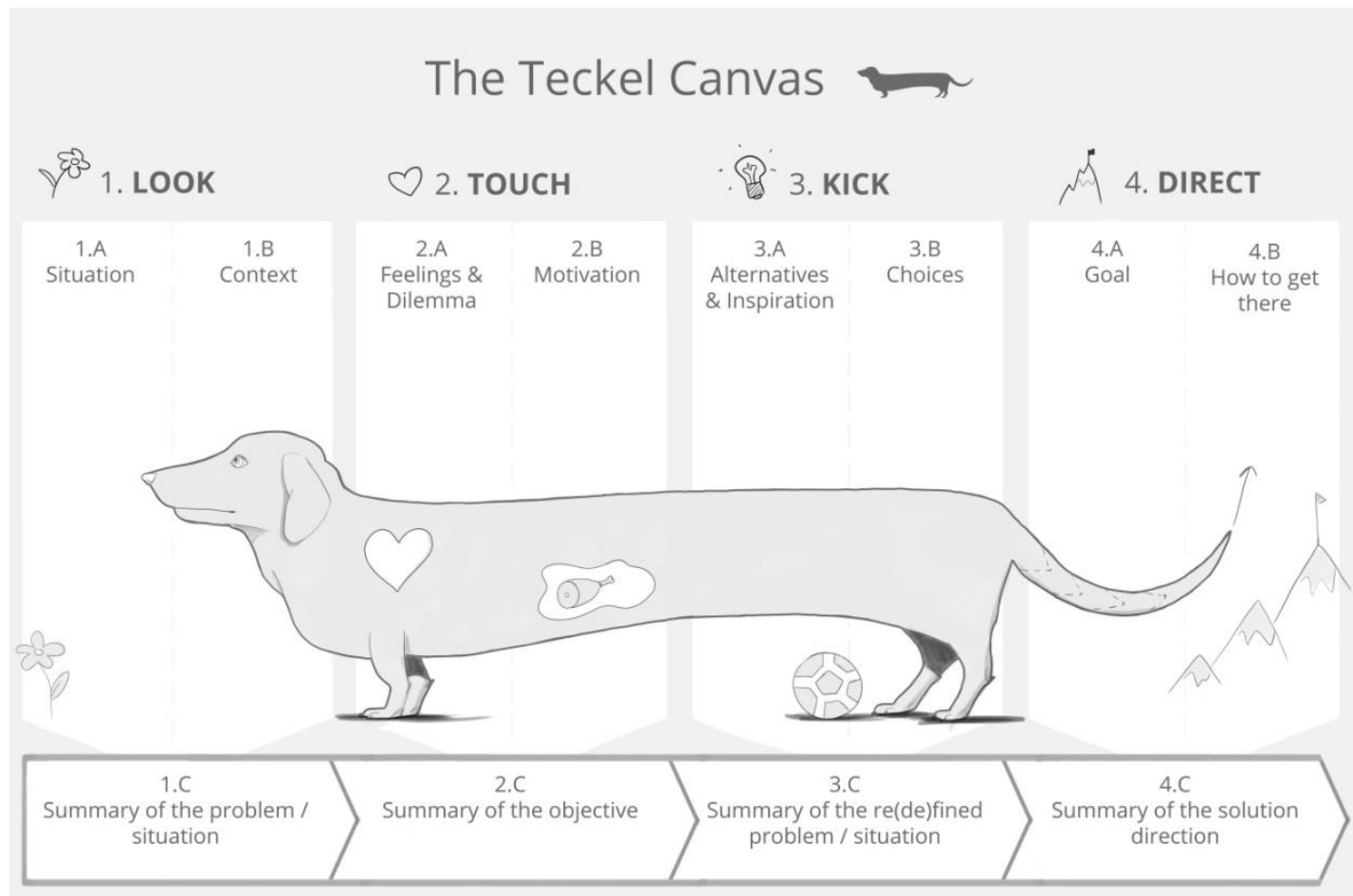

Figure 2. The Teckel reframing canvas

The eight sub-steps (under Look, Touch, Kick and Direct) cover the entire reframing process.

1.A Situation: asks to deconstruct the problem or the reason of doing the Teckel canvas.

1.B Context: follows up on the "situation", by exploring the deeper components that influence the situation, such as stakeholders, details or infrastructure.

1.C Summary of the problem: the goal here is to synthesise what was explored in 1.A and 1.B, summarised as "[Who] has the problem of $[\ldots]$ in [the situation] because $[\ldots]$ ".

2.A Feelings \& Dilemmas: here the team is invited to explore which emotions may cause the problem(s); the reactions and emotions that are caused by the problem(s); and the reason why the 
problem(s) might be hard to solve. This step does not only include emotional values, but also involves dilemmas surrounding technical, economic or social issues.

2.B Motivation: This step acknowledges the importance of intuition, by inquiring on the motivation of the stakeholders (e.g., clients of the company) or the company's own "gut-feeling".

2.C Summary of the objective: Again, it is important to encapsulate what was discussed above, by summarising why the team thinks there is a problem, why it is difficult and what they think it is important to address it.

3.A Alternatives \& inspiration: As a way to provide possible directions, this step explores how (closely or distantly) related problem(s) have been solved or get inspiration from products, services or approaches that the team is passionate about. This step allows the proposal of multiple directions, which could lead to reframing

3.B Choices: This step asks for the most important insight(s) and what needs to be solved in the shortand long-term, to makes choices about what the solution direction should entail. The client's team and facilitator decide together if the results have enough quality to continue with. If not, different tools (such as mentioned in figure 3) can help to tackle the remaining issues from a different perspective or in a more precise manner.

3.C Summary of the redefined problem: After the steps taken so far, the team can now outline what the refined/redefined problem is and how the perspective towards the problem might have changed.

4.A Goal: With a potentially reframed problem at hand, the team can draft one goal, which corresponds to concrete and abstract accomplishments that need to be achieved.

4.B How to get there: This step answers what is needed to get to the goal and what can be done now, tomorrow or in later steps.

4.C Summary of the solution direction: To complete the reframing approach, it is important to summarise concrete steps on what needs to be achieved and how it should be accomplished.

As the Teckel canvas is a framework that both explains and structures reframing, a set of cards was also developed to pragmatically support the canvas (Figure 3 ). The reframing tool cards, which portray well-known design techniques (such as personas or storytelling), were created to cater for each of the eight sub-steps with practical guidelines. These are not necessary to use with the canvas, but could still be a valuable addition, to enrich or enlarge the potential of the reframing facilitations sessions.

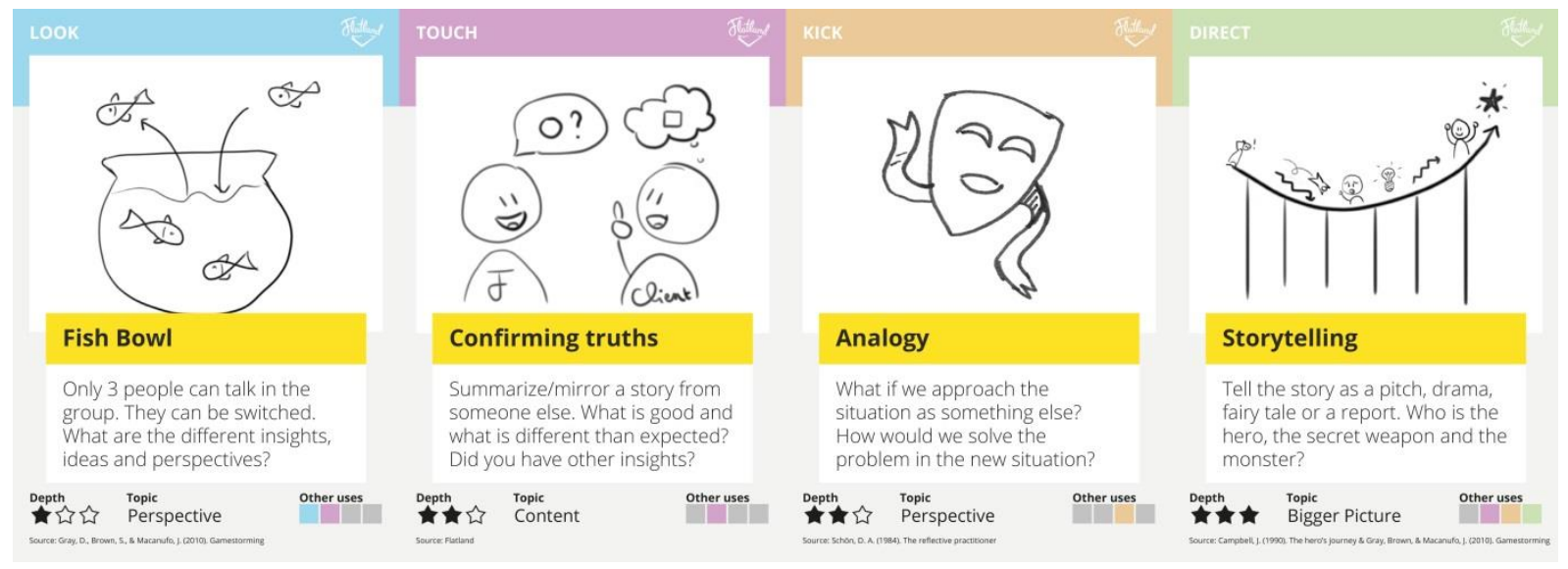

Figure 3. Four examples of the tool cards that were part of the bigger reframing project categorised on the four stages of the Teckel canvas. The colours refer to the four stages.

\section{RESULTS}

The final Teckel canvas was tested with two cases with real clients, each in sessions of four hours. The first case was facilitated by two experienced designers of Flatland, who were somewhat familiar with the Teckel canvas, while the second case was facilitated by the first author and creator of the Teckel canvas. In these two cases, parts of the Teckel canvas were slightly adapted to fit specific needs of the clients or to make the most of the sessions. To collect the data on the evaluation of the Teckel canvas, the facilitation sessions were observed, and combined with interviews with the facilitators (in case 1). The goal of the recurrent interviews with the professional facilitators of Flatland was to assess the canvas' explicitness, clarity and visual support and whether it could fit their current practices. 


\subsection{Case 1: Debt counselling low-literate people}

Flatland was approached by clients on the topic of debt counselling for low-literate people. The clients wanted a new way of recognising low-literacy among their customers but did not want a standard "informational booklet". Two experienced facilitators used the Teckel Canvas to facilitate the session. Considering the rather explorative problem, the Teckel Canvas was adjusted so that at the end of every stage, the problem space would be covered by many "How might we..." type of questions, rather than having just one goal. Next to that, the facilitation team wanted to give an extra dimension to the first two categories (Look and Touch) by forming different personas. In this way, the mixed situations, context, and conflicting feelings had to be filled in the Teckel Canvas in a structured manner to support moving to the next stages, see figure 4.

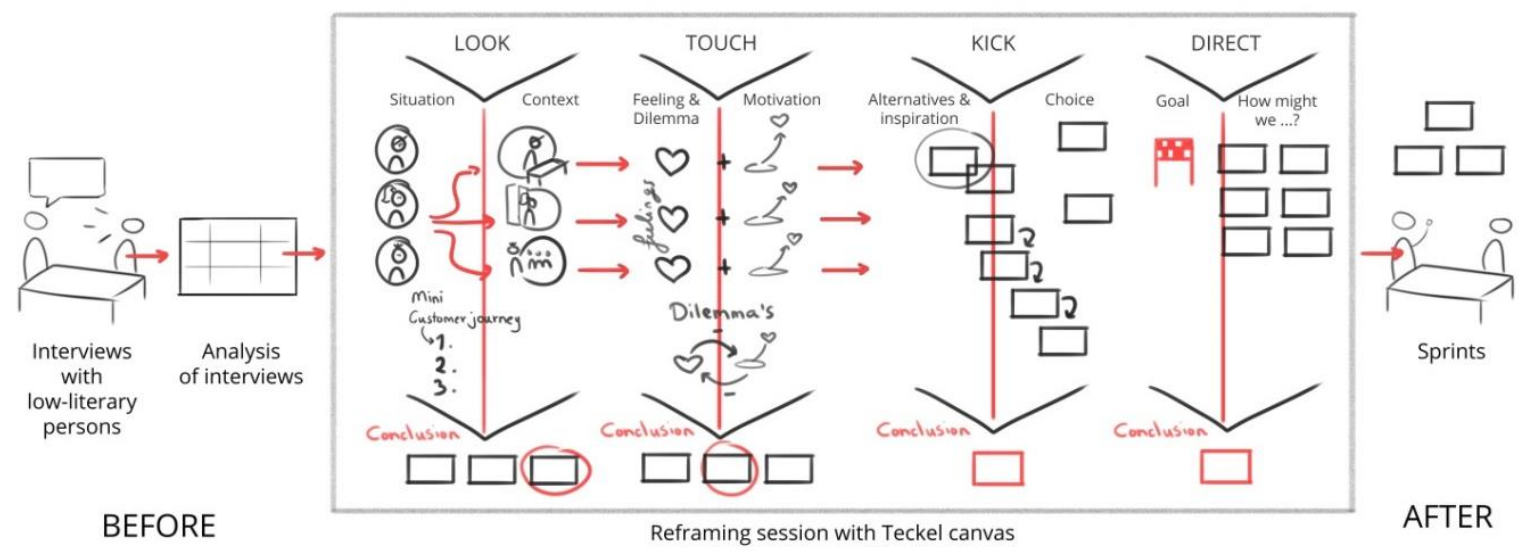

Figure 4. A visual representation of how the Teckel canvas was filled in for case 1, including how the three different personas were used in the Teckel canvas

The facilitators found the explicit nature of the stages, particularly the Touch and Kick ones, and how it provided nuances to their insights, very beneficial. Moreover, they thought that the driving towards a conclusive point after each phase rather useful in their session. It gave them a feeling of thoroughness in the reframing process. This left room for creative and participatory thinking together with the client. The client was very happy with the Teckel canvas because they were able to participate together at the same level, as problem owners and problem solvers, and had a complete overview of what was happening during the facilitation session. This reframing canvas did lead to multiple new goals or directions that the company and the client further worked on in the next sessions. Therefore, they were able to reframe the initial problem and move on to a plurality of new insights and directions.

\subsection{Case 2: Children day-care internal training centre}

A children day-care centre had to convince the board of commissioners to allow a new institution to be installed. Thus far, they have been hiring external parties to educate their pedagogical employees but wished to internalise education for the day-care' pedagogical employees. In this second case, it was not possible to reframe the day-care centre's problem definition of a new institution and how should it be set (which we could refer as the 'message'), but they did allow to reframe the way the message was portrayed (the 'medium'). More specifically, they needed to reframe how they communicated, because they knew they had to portray the emotional benefits for stakeholders to be able to convince the board of their plan. There were multiple stakeholders to consider, who would all look at the same outcome explaining the day-care plan. Two sessions were carried out, one using the reframing approach of the Teckel canvas and another to evaluate the outcome. An initial interview was held with a pedagogical employee to find the emotional burdens the current situation may carry. Furthermore, this first interview allowed an exploration of what could be the starting point of a final visualisation, which should portray the current emotional burdens and the possible direction to tackle them. This visualisation would be shown to stakeholders, as a result of the reframing process. After the first interview, the reframing canvas was used in the same manner as in case 1. Nevertheless, contrary to case 1, after each stage (Look, Touch, Kick and Direct), a sketch was created rather than a summarising sentence (figure 5). 
After the facilitation session with the Teckel canvas, the day-care centre was able to reframe the way they would communicate their new plan to stakeholders, using visual metaphors. The metaphors and accompanying explanations were used to understand the latent knowledge contextualising the problem and, more importantly, made it explicit to the children day-care centre and their stakeholders.

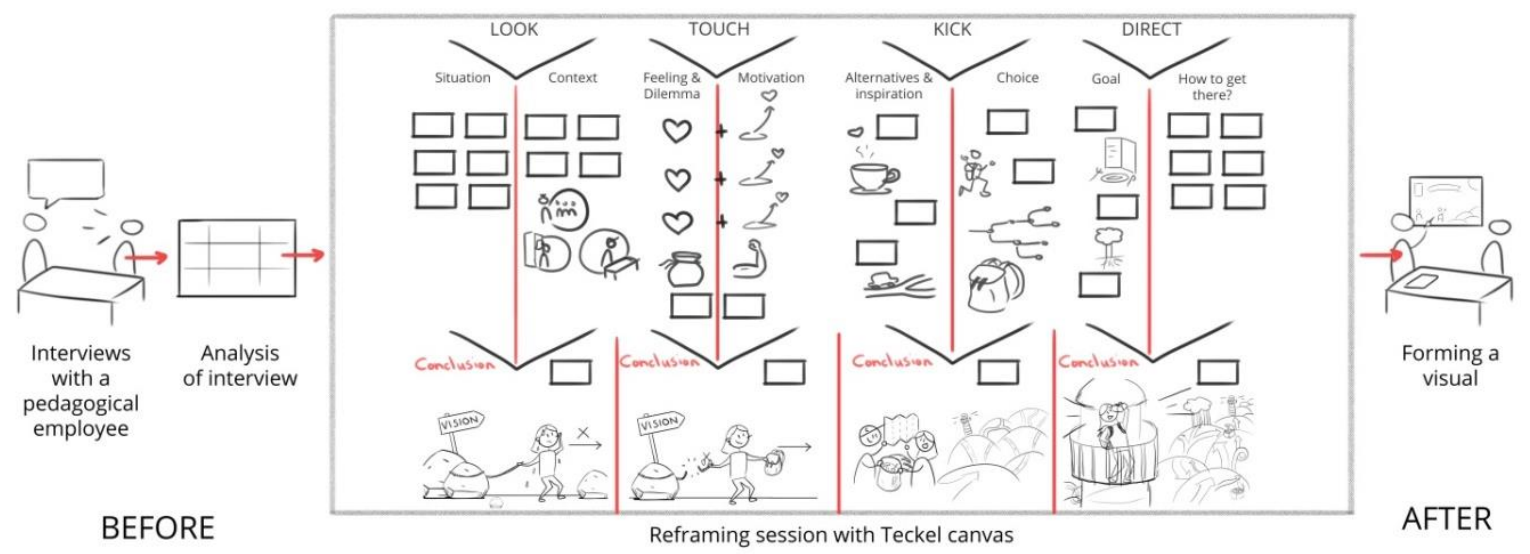

Figure 5. The filled in reframing canvas of the session of case 2

The outcome was a visual overview, which synthesised the insights and values discussed in the first session. The second session was focussed on the evaluation and iteration of the visualisation (figure 6). When assessing the Teckel canvas, the clients particularly valued the overview of all the different aspects in the reframing canvas, how it catered for emotional aspects and values and how the canvas urged them to summarise insights as visual metaphors.

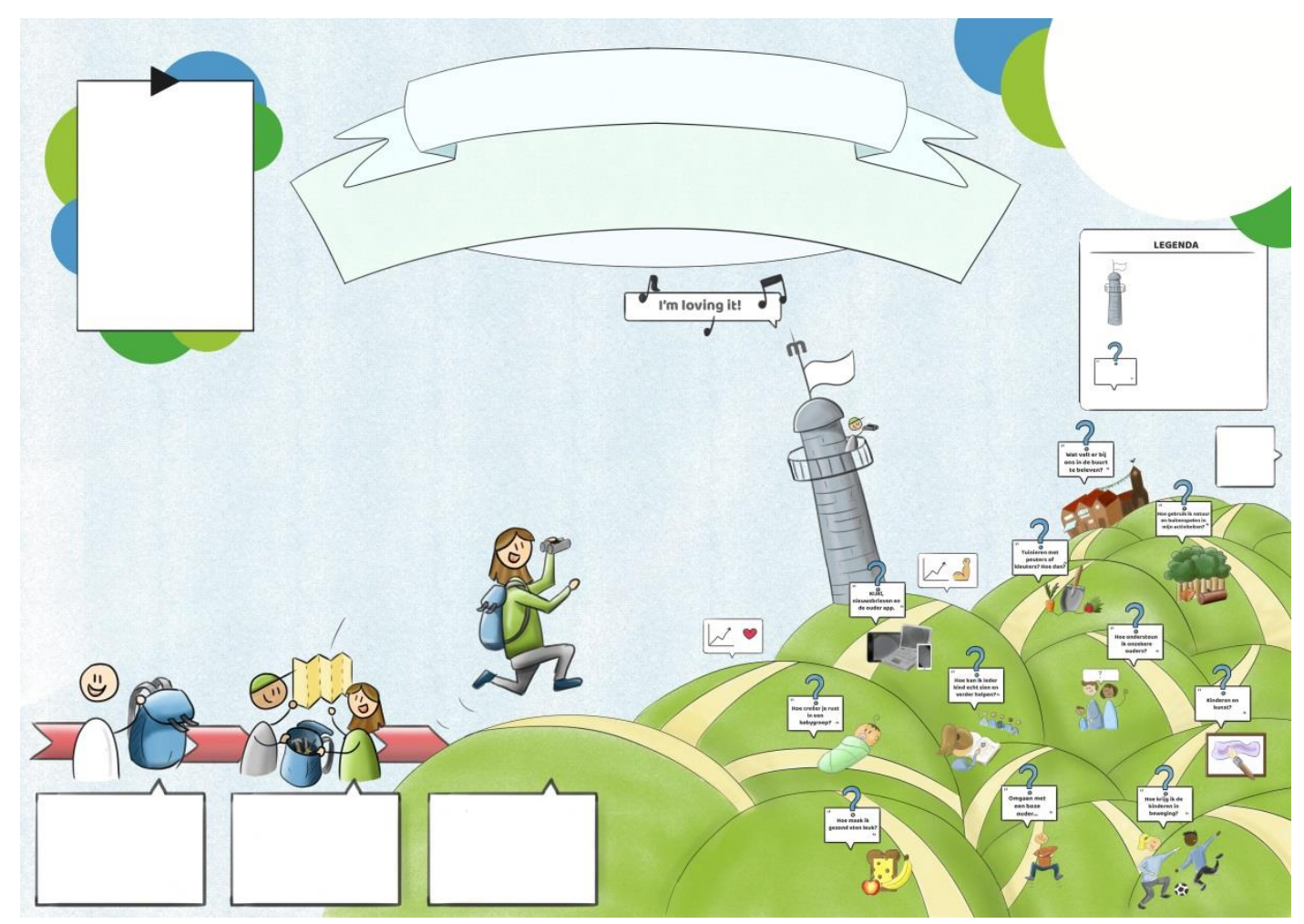

Figure 6. The final visualisation for the children day care centre that informs the stakeholders about their plans of the new institution

\section{DIscussion}

One of the major insights we collected was that the Teckel canvas is as its best when facilitators feel free to change the canvas or the way to work with it, to fit the clients' or the facilitator's needs. The 
Teckel canvas provides a meta-overview of the reframing process and can therefore be used as guidance, checklist or directly as it is, with variable levels of flexibility. It can be used with the reframing tool cards (figure 3) or with other existing tools or exercises preferred by the facilitators. The Teckel canvas adds explicitness in consulting and facilitation, both for facilitator and clients. It can provide freedom to the facilitator, as less effort is spent in structuring and more attention can be given to the quality of reframing and the guidance of the client in a creative and participatory session. Next to that, the problem and solution spaces are given equal attention, turning it into a more iterative process. The clients are involved in tackling the problem together, making the team feel they are deciding and accomplishing the change themselves (Sanden and Flipse, 2016; Wenger, 2000). This collaboration in facilitation increases the change of implementation and success rate because of created ownership by participation (Calabretta et al., 2016). Uncertainty, misconceptions and individual frames are made explicit to improve creativity and communication in a new frame (Sanden and Flipse, 2016). The two different cases show that reframing can be a tool for change; to alter not only the message but also the medium. More specifically, the Teckel canvas enables to change both the medium (the way information is communicated and visualised) and the message (problem and solution reframed, by providing new insights on the problem and/or solution directions). Similarly, the reframed outcome becomes more tangible and accessible through a visual medium, enabling new interpretations. In both cases, the facilitators and clients reported that the canvas helped them to better understand the context, problem and solution, and what should be conveyed in the final visualisation. As we could see in Case 2, reframing the medium is an approach that can still help the company when the facilitator is not allowed to reframe the message. This has been seen in practice; there is not always room for the facilitator to reframe how a client addresses the problem, by taking a step back onto the problem definition first proposed by the client (Flatland, 2018a; Owens, 2016). Often clients want an immediate solution to their problem, and are not willing to change their own perspective, way of work or the plan that they already came up with. Borrowing the useful metaphor of Aguirre et al. (2017), in which the facilitation process is compared to a synchronic flock of birds, the facilitator needs to enable the emergence of latent problems. The facilitator is both a participant and an active element of the "flock" to reveal patterns, which are yet undiscovered. When clients want to rush towards a solution, it is the responsibility of the facilitator to open up new directions and nudge the "flock" (the facilitation members) into discovering underlying issues.

\section{CONCLUSION}

As introduced earlier, the research question that guided this study was: How can we support problem reframing in facilitation sessions, in order to achieve reflective, explicit and visual outcomes in reframing? In this study, we presented the development of the Teckel canvas, created to illustrate and guide the reframing process as a practical visual tool during facilitation in practice. We evaluated its usefulness in two case studies and reflected on learned lessons. A number of caveats needs to be considered regarding this study. One possible limitation was that the final canvas was only tested with two cases, and those required minor changes to improve the use of the canvas in the sessions. This should be improved in the future, by investigating the canvas' usefulness in more cases. The Teckel canvas provided an overview of the reframing process and its stages, synthesised from a literature review of eight prominent reframing methods and approaches in the field of design and facilitation. However, future studies should investigate whether the identified reframing stages and sub-steps are sufficiently covered or whether all of them are needed in every reframing session. It is also recommended to investigate more in detail the usefulness of the reframing tool cards and other creative techniques in relation to the canvas. The cards were not included in the scope of this paper, so it is not yet clear which tools should be included or removed. In this study, we also did not specifically analyse the influence of the facilitator or designer during the reframing process, but this could be a major component in the effectiveness of the reframing method. The role of the facilitator is mostly unexplored in literature, let alone the influence of the facilitator in reframing sessions. Personal differences in a group can have a great influence on the facilitation team members and, subsequently, on the impact of the session. It is thus important to investigate the role of the facilitator in more detail, to understand how to navigate the reluctant voices in a group during a facilitation session. It is also important to realise that reframing may not lead to success at all times. Reframing is not the holy grail of design, and there are situations where it may actually lead to failure (Vermaas, Dorst, and 
Thurgood, 2015). Such situations could include when designers ignore the original problem of the client when solving the reformulated one or when the reframed problem is not accepted by the client (Vermaas, Dorst, and Thurgood, 2015). Thus, careful reflection integrated in this approach is necessary.

The findings of this study have a number of implications for future practice and education. For design practice, the Teckel canvas offers guidance and overview of the reframing process for facilitators to solve the complex problems of today. As demonstrated in this study, the Teckel canvas structures the ongoing process of reframing and offers tools to support reflection and explicitness. For education, it is important to consider how our design students are learning how to frame and reframe a given problem. Countless methods have been developed that focus on solution ideation. However, an implication of our work is that both reframing perspectives should be considered, focusing on the solution but also on the problem and latent aspects. To conclude, adding explicitness to reframing can benefit the exploration of complex problems, the design process and the solutions that are created with it to be more explicit, visual and reflective. The tangible guidance of the Teckel canvas can potentially help designers and facilitators during this process and helps to communicate reframing to their clients.

\section{REFERENCES}

Aguirre, M., Agudelo, N. and Romm, J. (2017), “Design Facilitation as Emerging Practice: Analyzing How Designers Support Multi-stakeholder Co-creation". She Ji: The Journal of Design, Economics, and Innovation, Vol. 3, No. 3, pp. 198-209.

Benammar, K. (2012), Reframing: The art of thinking differently. Boom uitgevers, Amsterdam.

Calabretta, G., Gemser, G. and Karpen, I. (2016), Strategic Design: Eight Essential Practices Every Strategic Designer Must Master, BIS Publishers, Amsterdam.

Flatland, (2018a), Data retrieved from interviews with the staff from Flatland

Flatland, (2018b), retrieved from https://flatland.agency/legacy-jongens-van-de-tekeningen/

Hey, J. H., C. K. Joyce and S. L. Beckman (2007), "Framing innovation: negotiating shared frames during early design phases." Journal of Design Research, Vol. 6 No. 1-2: pp. 79-99.

Hekkert, P. and Van Dijk, M. (2011). ViP-Vision in Design: A Guidebook for Innovators. BIS Publishers, Amsterdam

Maher, M. L., Poon, J. and Boulanger, S. (1996), "Formalising design exploration as co-evolution". In Advances in formal design methods for CAD (pp. 3-30). Springer, Boston, MA.

Maister, D. H., Green, C. H. and Galford, R. M. (2000), The trusted advisor. Simon and Schuster, London

Owens C. (2016), "How to get past just telling people to change their behaviour". [Weblog] Retrieved 7 December 2018, from https://xblog.xplane.com/how-to-get-past-just-telling-people-to-change-theirbehavior

Paton, B. and Dorst, K. (2011), "Briefing and reframing: A situated practice”. Design Studies, Vol. 32, No. 6, pp. 573-587.

Van der Sanden, M. C. and Flipse, S. M. (2016), "Science communication for uncertain science and innovation". Journal of Science Communication, Vol. 15, No. 06, pp. C05.

Schön, D. A. (1983), The reflective practitioner: how professionals think in action (Vol. 1). Basic Books, New York.

Stompff, G., Smulders, F. and Henze, L. (2016), "Surprises are the benefits: reframing in multidisciplinary design teams". Design Studies, Vol. 47, pp. 187-214.

Van Boeijen, A., Daalhuizen, J., van der Schoor, R. and Zijlstra, J. (2014), Delft design guide: Design strategies and methods. BIS Publishers, Amsterdam.

Vermaas, P., Dorst, C. and Thurgood, C. (2015), "Framing in design: A formal analysis and failure modes". Proceedings of the 20th International Conference on Engineering Design (ICED15), (July), 1-10.

Wiltschnig, S., Christensen, B. T. and Ball, L. J. (2013), "Collaborative problem-solution co-evolution in creative design". Design Studies, Vol. 34, No. 5, pp. 515-542.

Wedell-Wedellsborg, T. (2017), “Are you solving the right problems?" Harvard Business Review, Vol. 95, No. 1 , pp. 76-83.

Wenger, E. (2000), "Communities of practice and social learning systems". Organization, Vol. 7, No.2, pp. 225-246.

Wiltschnig, S., Christensen, B. T. and Ball, L. J. (2013), "Collaborative problem-solution co-evolution in creative design". Design Studies, Vol. 34, No. 5, pp. 515-542. 